\title{
On minimal additive complements of integers
}

\author{
Sándor Z. Kiss,${ }^{1 *}$ Csaba Sándor, ${ }^{1 \dagger}$ and Quan-Hui Yang $2 \ddagger$
}

1. Institute of Mathematics, Budapest University of Technology and Economics, H-1529 B.O. Box, Hungary

2. School of Mathematics and Statistics, Nanjing University of Information Science and Technology, Nanjing 210044, China

\begin{abstract}
Let $C, W \subseteq \mathbb{Z}$. If $C+W=\mathbb{Z}$, then the set $C$ is called an additive complement to $W$ in $\mathbb{Z}$. If no proper subset of $C$ is an additive complement to $W$, then $C$ is called a minimal additive complement. Let $X \subseteq \mathbb{N}$. If there exists a positive integer $T$ such that $x+T \in X$ for all sufficiently large integers $x \in X$, then we call $X$ eventually periodic. In this paper, we study the existence of a minimal complement to $W$ when $W$ is eventually periodic or not. This partially answers a problem of Nathanson.
\end{abstract}

*Email: kisspest@cs.elte.hu. This research was supported by the National Research, Development and Innovation Office NKFIH Grant No. K115288 and K109789 and K129335. This paper was supported by the János Bolyai Research Scholarship of the Hungarian Academy of Sciences. Supported by the ÚNKP-18-4 New National Excellence Program of the Ministry of Human Capacities.

†Email: csandor@math.bme.hu. This author was supported by the NKFIH Grant No. K109789 and K129335. This paper was supported by the János Bolyai Research Scholarship of the Hungarian Academy of Sciences.

${ }_{\ddagger}^{\ddagger}$ Email: yangquanhui01@163.com. This author was supported by the National Natural Science Foundation for Youth of China, Grant No. 11501299, the Natural Science Foundation of Jiangsu Province, Grant Nos. BK20150889, 15KJB110014 and the Startup Foundation for Introducing Talent of NUIST, Grant No. 2014r029. 
2010 Mathematics Subject Classifications: Primary 11B13, $11 \mathrm{~B} 34$.

Key words and phrases: Additive complements, minimal complements, periodic sets.

\section{Introduction}

Let $\mathbb{N}$ denote the set of nonnegative integers and $\mathbb{Z}$ be the set of integers. For $A, B \subseteq \mathbb{Z}$ and $k \in \mathbb{Z}$, let $A+B=\{a+b: a \in A, b \in B\}$ and $k A=\{k a: a \in A\}$. If $A+B=\mathbb{Z}$, then $A$ is called an additive complement to $B$ in $\mathbb{Z}$. If no proper subset of $A$ is a complement to $B$, then $A$ is called a minimal complement to $B$ in $\mathbb{Z}$.

It is easy to see that if $A \subseteq \mathbb{Z}$ is a (minimal) complement to $B \subseteq \mathbb{Z}$, then $A$ is also a (minimal) complement to $B+d, d \in \mathbb{Z}$, where $B+d=\{b+d$ : $b \in B\}$.

In 2011, Nathanson [4] proved the following theorem.

Nathanson's theorem (See [4, Theorem 8]). Let $W$ be a nonempty, finite set of integers. In $\mathbb{Z}$, every complement to $W$ contains a minimal complement to $W$.

In the same paper, Nathanson also posed the following problem.

Problem (See [4, Problem 11]). Let $W$ be an infinite set of integers. Does there exist a minimal complement to $W$ ? Does there exist a complement to $W$ that does not contain a minimal complement?

For the second part of the above problem, in 2012, Chen and Yang [2] gave two infinite sets $W_{1}$ and $W_{2}$ of integers such that there exists a complement to $W_{1}$ that does not contain a minimal complement and every complement to $W_{2}$ contains a minimal complement.

For the first part of the above problem, in 2012, Chen and Yang [2] proved the following results.

Theorem A (See [2, Theorem 1]). Let $W$ be a set of integers with inf $W=$ $-\infty$ and $\sup W=+\infty$. Then there exists a minimal complement to $W$. 
By Theorem A, now we only need to consider the cases inf $W>-\infty$ or $\sup W<+\infty$. Without loss of generality, we may assume that inf $W>-\infty$. Theorem B (See [2, Theorem 2]). Let $W=\left\{1=w_{1}<w_{2}<\cdots\right\}$ be a set of integers and

$$
\bar{W}=(\mathbb{Z} \cap(0,+\infty)) \backslash W=\left\{\overline{w_{1}}<\overline{w_{2}}<\cdots\right\}
$$

(a) If $\lim \sup _{i \rightarrow+\infty}\left(w_{i+1}-w_{i}\right)=+\infty$, then there exists a minimal complement to $W$.

(b) If $\lim _{i \rightarrow+\infty}\left(\overline{w_{i+1}}-\overline{w_{i}}\right)=+\infty$, then there does not exist a minimal complement to $W$.

Let $W=\cup_{k=0}^{\infty}\left[10^{k}, 2 \times 10^{k}\right]$. Then it is clear that both $\lim \sup _{i \rightarrow+\infty}\left(w_{i+1}-\right.$ $\left.w_{i}\right)=+\infty$ and $\lim \sup _{i \rightarrow+\infty}\left(\overline{w_{i+1}}-\overline{w_{i}}\right)=+\infty$ hold. Hence $\lim _{i \rightarrow+\infty}\left(\overline{w_{i+1}}-\right.$ $\left.\overline{w_{i}}\right)=+\infty$ in Theorem B (b) cannot be changed to $\lim \sup _{i \rightarrow+\infty}\left(\overline{w_{i+1}}-\overline{w_{i}}\right)=$ $+\infty$.

In this paper, we will give further results on Nathanson's problem and deal with some sets $W$ do not satisfy the conditions of Theorem B.

First we give some definitions. Let $S \subseteq \mathbb{N}$. Denote by $S \bmod m$ the set of residues of $S$ modulo $m$, i.e.,

$$
S \bmod m=\{r: r \in\{0,1, \ldots, m-1\}, r \equiv s(\bmod m) \text { for some } s \in S\} .
$$

Let $X \subseteq \mathbb{N}$. If there exists a positive integer $T$ such that $x+T \in X$ for all $x \in X$, then we call $X$ periodic with period $T$. If $X \cup C$ is a periodic set for some finite set $C \subseteq \mathbb{N}$, then we call $X$ quasiperiodic. If there exists a positive integer $T$ such that $x+T \in X$ for all sufficiently large integers $x \in X$, then we call $X$ eventually periodic with period $T$. Clearly, a periodic set must be quasiperiodic and a quasiperiodic set must be eventually periodic. If $W$ is eventually periodic with $|\mathbb{N} \backslash W|=+\infty$, then both $\lim _{i \rightarrow+\infty}\left(w_{i+1}-w_{i}\right)<$ $+\infty$ and $\lim _{i \rightarrow+\infty}\left(\overline{w_{i+1}}-\overline{w_{i}}\right)<+\infty$ hold. Hence $W$ does not satisfy the conditions of Theorem B.

Suppose that $W$ is an eventually periodic set and $m$ is a positive period. 
By shifting a number, we may assume that $W$ has the following structure:

$$
W=\left(m \mathbb{N}+X_{m}\right) \cup Y^{(0)} \cup Y^{(1)}
$$

where $X_{m} \subseteq\{0,1, \ldots, m-1\}, Y^{(0)} \subseteq \mathbb{Z}^{-}, Y^{(1)}$ are finite sets with $Y^{(0)} \bmod m \subseteq$ $X_{m}$ and $\left(Y^{(1)} \bmod m\right) \cap X_{m}=\emptyset$.

For example, if $W=\{2,4,7,8,9,12,13,17,18,22,23,27,28, \ldots\}$, then by shifting a number 5 , we may assume that

$$
W=\{-3,-1,2,3,4,7,8,12,13,17,18,22,23, \ldots\} .
$$

Hence $m=5, X_{m}=\{2,3\}, Y^{(0)}=\{-3\}, Y^{(1)}=\{-1,4\}$.

In this paper, we study that what conditions are needed to ensure the existence of a minimal complement to $W$. First we prove a sufficient condition.

Theorem 1. Let $W$ be defined in (1). If there exists a minimal complement to $W$, then there exists $C \subseteq\{0,1, \ldots, m-1\}$ such that the following two conditions hold:

(a) $C+\left(X_{m} \cup Y^{(1)}\right) \bmod m=\{0,1, \ldots, m-1\}$;

(b) For any $c \in C$, there exists $y \in Y^{(1)}$ such that

$c+y \not \equiv c^{\prime}+x(\bmod m)$, where $c^{\prime} \in C, x \in X_{m}$.

Remark 1. By the proof of Theorem 1, we know that Theorem 1 also holds when $Y^{(1)}$ is an infinite set with $\left|Y^{(1)} \cap \mathbb{Z}^{-}\right|<+\infty$.

Let $m=3, X_{m}=\{0\}, Y^{(1)} \subseteq 3 \mathbb{N}+1$. By Theorem 1, we have the following corollary.

Corollary 1. Let $Y \subseteq 3 \mathbb{N}+1$ and $W=3 \mathbb{N} \cup Y$. Then there does not exist a minimal complement to $W$.

Remark 2. We can choose an infinite set $Y$ in Corollary 1 such that $W$ is not eventually periodic. Hence, there exists an infinite, not eventually periodic set $W \subseteq \mathbb{N}$ such that $w_{i+1}-w_{i} \in\{1,2,3\}$ for all $i$, and there does not exist a minimal complement to $W$. 
Remark 3. If $W \subseteq \mathbb{N}$ is a quasiperiodic set, then $Y^{(1)}=\emptyset$ and the condition (b) in Theorem 1 does not hold. Hence there does not exist a minimal complement to $W$.

In the next step we prove a necessary condition.

Theorem 2. Let $W$ be defined in (1). Suppose that there exists $C \subseteq$ $\{0,1, \ldots, m-1\}$ such that the following two conditions hold:

(a) $C+\left(X_{m} \cup Y^{(1)}\right) \bmod m=\{0,1, \ldots, m-1\}$;

(b) For any $c \in C$, there exists $y \in Y^{(1)}$ such that $c+y \not \equiv c^{\prime}+x(\bmod m)$, where $c^{\prime} \in C \backslash\{c\}, x \in X_{m} \cup Y^{(1)}$.

Then there exists a minimal complement to $W$.

By Theorems 1 and 2, we have the following corollary.

Corollary 2. Let $W=\left(m \mathbb{N}+X_{m}\right) \cup Y^{(0)} \cup\{a\}$, where $X_{m} \subseteq\{0,1, \ldots, m-$ $1\},\left(Y^{(0)} \bmod m\right) \subseteq X_{m}$ and $a \not \equiv x(\bmod m)$ if $x \in X_{m}$. Then there exists a minimal complement to $W$ if and only if there exists a subset $C \subseteq$ $\{0,1, \ldots, m-1\}$ such that:

(a) $C+\left(X_{m} \cup\{a\}\right) \bmod m=\{0,1, \ldots, m-1\}$;

(b) For any $c \in C, c+a \not \equiv c^{\prime}+x(\bmod m)$, where $c^{\prime} \in C \backslash\{c\}$ and $x \in X_{m}$.

We see that Theorems 1 and 2 transfer Nathanson's problem into a finite modulo version when $W$ is an eventually periodic set. In the next theorem, we give a sufficient and necessary condition, but we cannot bound the module.

Theorem 3. Let $W$ be defined in (1). There exists a minimal complement to $W$ if and only if there exists $T \in \mathbb{Z}^{+}, m \mid T$, and $C \subseteq\{0,1, \ldots, T-1\}$ such that

(a) $C+\left(X_{T} \cup Y^{(1)}\right) \bmod T=\{0,1, \ldots, T-1\}$, where $X_{T}=\cup_{i=0}^{\frac{T}{m}-1}\{i m+$ $\left.X_{m}\right\}$;

(b) for any $c \in C$, there exists $y \in Y^{(1)}$ for which $c+y \not \equiv c^{\prime}+x(\bmod T)$, where $c^{\prime} \in C \backslash\{c\}$ and $x \in X_{T}$. 
Finally, as a complement to Remark 2, we give the following theorem.

Theorem 4. There exists an infinite, not eventually periodic set $W \subseteq \mathbb{N}$ such that $w_{i+1}-w_{i} \in\{1,2\}$ for all $i$ and there exists a minimal complement to $W$.

Now we pose two problems for further research.

Problem 1. We know that Theorem 1 also holds when $Y^{(1)}$ is infinite. Is Theorem 2 also true when $Y^{(1)}$ is infinite?

Problem 2. Does there exist an upper bound for $T$ in Theorem 3 using $m, Y^{(0)}$ and $Y^{(1)}$ ?

\section{Proofs}

Proof of Theorem 1. Suppose that $D$ is a minimal complement to $W$. For $i \in\{0,1, \ldots, m-1\}$, let $D_{i}=\{d \in D: d \equiv i(\bmod m)\}$ and

$$
C=\left\{j: 0 \leq j \leq m-1 \text { and }\left|D_{j} \cap \mathbb{Z}^{-}\right|=+\infty\right\}
$$

For any $t \in\{0,1, \ldots, m-1\} \backslash C$, the set $\{d \in D: d \equiv t(\bmod m)\}+W$ does not contain any sufficiently small negative integers. It follows from $D+W=\mathbb{Z}$ that $C+W \bmod m=\{0,1, \ldots, m-1\}$. That is, $C+\left(X_{m} \cup\right.$ $\left.Y^{(1)}\right) \bmod m=\{0,1, \ldots, m-1\}$.

Next we shall prove (b). Suppose that there exists $c \in C$ such that for any $y \in Y^{(1)}$ there exist $c^{\prime} \in C$ and $x \in X_{m}$ with $c+y \equiv c^{\prime}+x(\bmod m)$. We take an integer $d \in D$ such that $d \equiv c(\bmod m)$ and we shall prove that $D \backslash\{d\}$ is also a complement to $W$. For any integer $n$, write $n=d^{\prime}+w$, where $d^{\prime} \in D$ and $w \in W$.

Case 1. $d^{\prime} \neq d$. Then $n=d^{\prime}+w \in(D \backslash\{d\})+W$.

Case 2. $d^{\prime}=d$.

Subcase 2.1. $(\{w\} \bmod m) \subseteq X_{m}$. In this case, there exists a positive integer $k_{0}$ such that $w+k m \in W$ for all integers $k \geq k_{0}$. Since $\left|D_{c} \cap \mathbb{Z}^{-}\right|=$ 
$+\infty$, it follows that there exists an integer $k \geq k_{0}$ such that $d-k m \in D$. Hence $n=(d-k m)+(w+k m)$, where $d-k m \in D \backslash\{d\}$ and $w+k m \in W$. That is, $n \in(D \backslash\{d\})+W$.

Subcase 2.2. $w \in Y^{(1)}$. Since $(\{c+y\} \bmod m) \subseteq\left(C+X_{m} \bmod m\right)$ for any $y \in Y^{(1)}$ and $d \equiv c(\bmod m), w \in Y^{(1)}$, it follows that $\{n\} \bmod m=$ $\{d+w\} \bmod m\} \subseteq\left(C+X_{m} \bmod m\right)$. Hence there exist a $c^{\prime} \in D$ with $c^{\prime}(\bmod m) \in C$ and $x \in W$ with $x \bmod m \in X_{m}$ such that $n \equiv c^{\prime}+$ $x(\bmod m)$. We choose a sufficiently large integer $k$ such that $c^{\prime}-k m \in$ $D, c^{\prime}-k m \neq d$ and $x+k m \in W$. Hence $n=\left(c^{\prime}-k m\right)+(x+k m)$, where $c^{\prime}-k m \in D \backslash\{d\}$ and $x+k m \in W$.

Hence, $(D \backslash\{d\})+W=\mathbb{Z}$ which contradicts the fact that $D$ is a minimal complement. Therefore, (b) holds.

Proof of Theorem 2. Let $C_{1}=C+X_{m} \bmod m, C_{2}=\{0,1, \ldots, m-1\} \backslash C_{1}$,

$$
\begin{aligned}
& C^{\prime}=\{d \in \mathbb{Z}: d \equiv c(\bmod m) \text { for some } c \in C\}, \\
& C_{1}^{\prime}=\left\{d \in \mathbb{Z}: d \equiv c(\bmod m) \text { for some } c \in C_{1}\right\}, \\
& C_{2}^{\prime}=\left\{d \in \mathbb{Z}: d \equiv c(\bmod m) \text { for some } c \in C_{2}\right\} .
\end{aligned}
$$

By (a), we have $C^{\prime}+W=\mathbb{Z}$. Since $C+X_{m} \bmod m=C_{1}$, it follows that

$$
C^{\prime}+\left(W \backslash Y^{(1)}\right) \bmod m=C+X_{m} \bmod m=C_{1},
$$

and so $\left(C^{\prime}+\left(W \backslash Y^{(1)}\right)\right) \cap C_{2}^{\prime}=\emptyset$. It follows from (b) that $C_{2}^{\prime} \neq \emptyset$. Noting that $C^{\prime}+W=\mathbb{Z}$, we have $\left(C^{\prime}+Y^{(1)} \bmod m\right) \supseteq C_{2}^{\prime}$. Since $Y^{(1)}$ is a finite set, by the proof of Nathanson's theorem (See [4, Theorem 4, page 2015]), there exists $D^{\prime} \subseteq C^{\prime}$ such that $D^{\prime}+Y^{(1)} \supseteq C_{2}^{\prime}$ and for any $d \in D^{\prime}$,

$$
\left(D^{\prime} \backslash\{d\}\right)+Y^{(1)} \nsupseteq C_{2}^{\prime}
$$

Next we shall prove that $D^{\prime}$ is a minimal complement to $W$.

For $i \in C$, let $D_{i}^{\prime}=\left\{d \in D^{\prime}: d \equiv i(\bmod m)\right\}$. First we prove that $\left|D_{i}^{\prime} \cap \mathbb{Z}^{-}\right|=+\infty$ for all $i \in C$. Suppose that there exists a $j \in C$ 
such that $\left|D_{j}^{\prime} \cap \mathbb{Z}^{-}\right|<+\infty$. By (b), there exists a $y \in Y^{(1)}$ such that $j+y \not \equiv c+x(\bmod m)$, where $c \in C \backslash\{j\}, x \in X_{m} \cup Y^{(1)}$ and so

$$
D^{\prime}+Y^{(1)} \nsupseteq\{d \in \mathbb{Z}: d \equiv j+y(\bmod m)\}
$$

Noting that $(\{j+y\} \bmod m) \nsubseteq C+X_{m} \bmod m=C_{1}$, we have $(\{j+$ y\} $\bmod m) \subseteq C_{2}$. It follows that $D^{\prime}+Y^{(1)} \nsupseteq C_{2}^{\prime}$, a contradiction. Hence, $\left|D_{i}^{\prime} \cap \mathbb{Z}^{-}\right|=+\infty$ for all $i \in C$.

Next we prove that $D^{\prime}$ is a complement. For any integer $n \in C_{1}^{\prime}$, by $C+X_{m} \bmod m=C_{1}$, there exists $c \in C$ and $x \in X_{m}$ such that $n \equiv c+$ $x(\bmod m)$. Since $\left|D_{c}^{\prime} \cap \mathbb{Z}^{-}\right|=+\infty$, there exists a sufficiently small negative integer $d \in D_{c}^{\prime}$ such that $n-d>0$. The congruences $n \equiv c+x(\bmod m)$ and $d \equiv c(\bmod m)$ imply that $n-d \equiv x(\bmod m)$. Hence, $n-d \in m \mathbb{N}+X_{m}$ and so

$$
n=d+(n-d) \in D_{c}^{\prime}+\left(m \mathbb{N}+X_{m}\right) \subseteq D^{\prime}+W .
$$

Hence $C_{1}^{\prime} \subseteq D^{\prime}+W$. On the other hand, $D^{\prime}+W \supseteq D^{\prime}+Y^{(1)} \supseteq C_{2}^{\prime}$. Therefore, $D^{\prime}+W=\mathbb{Z}$.

Finally, we prove that $D^{\prime}$ is a minimal complement. For any $d \in D^{\prime}$, we have

$$
\left(\left(D^{\prime} \backslash\{d\}\right)+\left(W \backslash Y^{(1)}\right) \bmod m\right) \subseteq C+X_{m} \bmod m=C_{1}
$$

It follows that

$$
\left(\left(D^{\prime} \backslash\{d\}\right)+\left(W \backslash Y^{(1)}\right)\right) \cap C_{2}^{\prime}=\emptyset,
$$

and so $\left(D^{\prime} \backslash\{d\}\right)+W \nsupseteq C_{2}^{\prime}$. Hence $\left(D^{\prime} \backslash\{d\}\right)+W \neq \mathbb{Z}$.

Therefore, $D^{\prime}$ is a minimal complement to $W$.

Proof of Theorem 3. Assume that the set $W$ satisfies the conditions of Theorem 3. Applying Theorem 2 with $m=T$, it follows that $W$ has a minimal complement.

Suppose that $W$ has a minimal complement denoted by $E$. We will prove the existence of a positive integer $T$ and a set $C \subseteq\{0,1, \ldots, T-1\}$ which satisfy the conditions of Theorem 3 . We will show that there exist positive 
integers $K$ and $L$ with $L>K$ such that $T=L-K$. We will prove that this integer $T$ and the set

$$
C=\{l: K \leq l<L, \quad l \in E\} \bmod L-K
$$

are suitable.

For $0 \leq i<m$, let

$$
E_{i}^{-}=\{e: e<0, e \in E, e \equiv i(\bmod m)\}
$$

Let $0 \leq i_{1}<i_{2}<\ldots<i_{t}<m$ be the sequence of indices with $\left|E_{i_{j}}^{-}\right|=\infty$. It is clear that there exists an integer $N_{0}$ such that, $e \in E$ and $e \leq N_{0}$ imply that $e \in E_{i_{j}}$ for some $i_{j}$. It follows from Theorem 1 that $Y^{(0)} \cup Y^{(1)} \neq \emptyset$. Let

$$
\begin{aligned}
& y_{+}=\max \left\{y: y \in Y^{(0)} \cup Y^{(1)}\right\}, \\
& y_{-}=\min \left\{y: y \in Y^{(0)} \cup Y^{(1)}\right\},
\end{aligned}
$$

and $y_{0}=y_{+}-y_{-}+1$. Let $\chi_{E}(k)$ denote the characteristic function of the set $E$, i.e.,

$$
\chi_{E}(k)=\left\{\begin{array}{l}
1, \text { if } k \in E ; \\
0, \text { if } k \notin E .
\end{array}\right.
$$

Define the positive integer $A$ by $A=N_{0}+\min \left\{0, y_{-}\right\}$. Consider the following vectors:

$$
\begin{aligned}
\mathbf{v}_{A} & =\left(\chi_{E}\left(A+y_{-}\right), \chi_{E}\left(A+y_{-}+1\right), \ldots, \chi_{E}\left(A+y_{+}\right)\right) \\
\mathbf{v}_{A-m} & =\left(\chi_{E}\left(A-m+y_{-}\right), \chi_{E}\left(A-m+y_{-}+1\right), \ldots, \chi_{E}\left(A-m+y_{+}\right)\right), \\
& \vdots \\
\mathbf{v}_{A-i m} & =\left(\chi_{E}\left(A-i m+y_{-}\right), \chi_{E}\left(A-i m+y_{-}+1\right), \ldots, \chi_{E}\left(A-i m+y_{+}\right)\right),
\end{aligned}
$$

It is clear that there are infinitely many vectors $\mathbf{v}_{A}, \mathbf{v}_{A-m}, \ldots, \mathbf{v}_{A-i m}, \ldots$, each of them has $y_{0}$ coordinates, which are 0 or 1 . Since there are at most $2^{y_{0}}$ different vectors, by the pigeon hole principle, there exists a vector $\mathbf{v}$ 
among them which occurs infinitely many times. In other words there exists an infinite sequence $0 \leq k_{1}<k_{2}<\cdots$ such that $\mathbf{v}_{A-k_{i} m}=\mathbf{v}$. Define $L$ by $L=A-k_{1} m$. Obviously it can be chosen a $k_{i}$ large enough such that

$$
\left[A-k_{i} m, A-k_{1} m\left[\cap E_{i_{j}} \neq \emptyset\right.\right.
$$

and $k_{i} m-k_{1} m \geq \max \left\{y_{0}, y_{+},-y_{-}\right\}$hold for every index $i_{j}$. In view of this fact we define $K$ by $K=A-k_{i} m$. and $T=L-K$. It follows from the definition that $K$ and $L$ have the following properties.

$$
\begin{gathered}
L \leq N_{0}+\min \left\{0, y_{-}\right\}, \\
K \leq L-y_{0}, \\
m \mid L-K, \\
\chi_{E}(K+i)=\chi_{E}(L+i), \text { for } y_{-} \leq i \leq y_{+},
\end{gathered}
$$

In the next step we show that the positive integer $T$ and set $C$ defined above satisfy the conditions of Theorem 3 .

We know from the conditions of Theorem 3 that $W=\left(T \mathbb{N}+X_{T}\right) \cup$ $Y^{(0)} \cup Y^{(1)}$ and $X_{T} \subseteq\{0,1 \ldots, T-1\}$. First we prove that $C+\left(X_{T} \cup\right.$ $\left.Y^{(1)}\right) \bmod T=\{0,1, \ldots T-1\}$. Let $K \leq l<L$. It follows that $l=e+w$, where $e \in E$ and $w \in W$. As $w \geq \min \left\{0, y_{-}\right\}$, it follows from (2) that $e=l-w<L-\min \left\{0, y_{-}\right\} \leq N_{0}$, thus we have $e \in E_{i_{j}}^{-}$.

Suppose that $w \in Y^{(0)} \cup Y^{(1)}$. Then we have $y_{-} \leq w \leq y_{+}$, which implies that $e=l-w$, where $K-y_{+} \leq e<L-y_{-}$. We have three cases.

Case 1. $K-y_{+} \leq e<K$. By (5) we have $e+(L-K) \in E$ and $K \leq L-y_{+} \leq e+L-K<L$. Thus we have $l \equiv c+w(\bmod T)$, where $c \in C$ and $w \in X_{T} \cup\left(Y^{(1)} \bmod T\right)$. 
Case 2. $K \leq e<L$. It follows that $l \equiv c+w(\bmod T)$, where $c \in C$ and $w \in X_{T} \cup\left(Y^{(1)} \bmod T\right)$.

Case 3. $L \leq e<L-y_{-}$. By (5) we have $e-(L-K) \in E$ and $K \leq e-(L-K)<K-y_{-}<L$, thus we have $l \equiv c+w(\bmod T)$, where $c \in C$ and $w \in X_{T} \cup\left(Y^{(1)} \bmod T\right)$.

Suppose that $w \in T \mathbb{N}+X_{T}$. Since $w \geq 0$ and $e=l-w<L \leq N_{0}$, we have $e \in E_{i_{j}}^{-}$, which implies that $e \equiv i_{j}(\bmod m)$. It follows from $(6)$ that there exists an $e^{\prime}$ such that $e^{\prime} \in E_{i_{j}}^{-}$and $K \leq e^{\prime}<L$, thus we have $e^{\prime} \equiv$ $i_{j}(\bmod m)$. Let $w \equiv x(\bmod m)$, where $0 \leq x<m, x \in X_{m}$. Obviously, $l \equiv e+w \equiv i_{j}+x \equiv e^{\prime}+x(\bmod m)$. By using $(4)$ it follows that there exists a $u$ with $0 \leq u<\frac{L-K}{m}$ such that $l \equiv e^{\prime}+u m+x(\bmod L-K)$. Therefore, $c \equiv e^{\prime}(\bmod L-K)$, where $0 \leq c<L-K, c \in C$ and $0 \leq u m+x<L-K$ and $u m+x \in X_{T}$ are suitable.

In the next step we show that the second condition of Theorem 3 holds. For every $K \leq e<L \leq N_{0}$ and $e \in E$, there exists a $w \in W$ such that $e+w \neq e^{\prime}+w^{\prime}$, when $e \neq e^{\prime}, e^{\prime} \in E$ and $w^{\prime} \in W$. If $w \in\left(m \mathbb{N}+X_{m}\right) \cup Y^{(0)}$, then there exists a positive integer $s$ such that $e+w=(e-s m)+(w+s m)$, where $e-s m \in E$ and $w+s m \in W$, which is absurd. Then we may assume that $w \in Y^{(1)}$. It follows that $K+y_{-} \leq e+w \leq L+y_{+}$. Obviously, it is enough to prove that $e+w \not \equiv e^{\prime}+w^{\prime}(\bmod L-K)$, where $K \leq e^{\prime}<L$, $e^{\prime} \in E$ and $w^{\prime} \in X_{T} \cup\left(Y^{(1)} \bmod T\right)$. Suppose that for $w^{\prime} \in X_{T}$ we have $e+w=e^{\prime}+w^{\prime}+t(L-K)$ for some integer $t$. Then we have $e+w=$ $e^{\prime}+w^{\prime}+t(L-K)=\left(e^{\prime}-s m\right)+\left(w^{\prime}+s m+t(L-K)\right)$, where $e^{\prime}-s m \in E$ and $w^{\prime}+s m+t(L-K) \in W$ for some positive integer $s$, which is absurd.

For any $w^{\prime} \in Y^{(1)}$, clearly we have $K+y_{-} \leq e+w, e^{\prime}+w^{\prime} \leq L+y_{+}$. Assume that $e+w \equiv e^{\prime}+w^{\prime}(\bmod L-K)$. It follows that either $e+w=e^{\prime}+w^{\prime}$ or $e+w=e^{\prime}+w^{\prime}+(L-K)$ or $e+w=e^{\prime}+w^{\prime}-(L-K)$.

Case 1. $e+w=e^{\prime}+w^{\prime}$. Then we have $e=e^{\prime}$ and $w=w^{\prime}$, which is impossible.

Case 2. $e+w=e^{\prime}+w^{\prime}+(L-K)$. Then we have $K+y_{-} \leq e^{\prime}+w^{\prime} \leq K+y_{+}$. 
Thus we have $K+y_{-}-y_{+} \leq e^{\prime} \leq K+y_{+}-y_{-}$. It follows from (5) that $e^{\prime}+(L-K) \in E$ which implies that $e+w=\left(\left(e^{\prime}+(L-K)\right)+w^{\prime}\right.$. Therefore we have $w=w^{\prime}$ and $e=e^{\prime}+(L-K)$ which is absurd because $K \leq e, e^{\prime}<L$.

Case 3. $e+w=e^{\prime}+w^{\prime}-(L-K)$. Then we have $L+y_{-} \leq e^{\prime}+w^{\prime} \leq L+y_{+}$. Thus we have $L+y_{-}-y_{+} \leq e^{\prime} \leq L+y_{+}-y_{-}$. It follows from (5) that $e^{\prime}-(L-K) \in E$ which implies that $e+w=\left(\left(e^{\prime}-(L-K)\right)+w^{\prime}\right.$. Therefore we have $w=w^{\prime}$ and $e=e^{\prime}-(L-K)$, which is a contradiction because $K \leq e, e^{\prime}<L$.

The proof of Theorem 3 is completed.

Proof of Theorem 4. By induction we can construct $\left\{d_{i}\right\}_{i=1}^{\infty},\left\{W_{i}\right\}_{i=1}^{\infty}$ and $\left\{c_{i}\right\}_{i=1}^{\infty}$ such that

(i) $d_{1}=-1, W_{1}=\{1,2, \ldots, 12\}, c_{1}=-3$;

(ii) $d_{i}$ is the largest negative integer $\notin W_{i-1}+\left\{c_{1}, c_{2}, \ldots, c_{i-1}\right\}$ for $i \geq 2$;

(iii) $c_{i}<d_{i}+2 c_{i-1}$ for all $i \geq 2$;

(iv) for $i \geq 2$, let $W_{i}=W_{i-1} \cup\left(\left[-2 c_{i-1},-2 c_{i}-1\right] \backslash \cup_{j=1}^{i-1}\left\{-c_{i}+d_{j}\right\}\right)$.

Let $W=\cup_{i=1}^{\infty} W_{i}$ and $C=\left\{c_{i}\right\}_{i=1}^{\infty}$.

Now we prove that $C$ is a minimal complement to $W$.

First we prove $d_{i+1}-d_{i} \leq-2$ for all integers $i \geq 1$. Clearly $d_{2}=$ $-3, d_{2}-d_{1}=-2$. Suppose that $d_{i+1}-d_{i} \leq-2$ for all integers $i<k(k \geq 2)$. Since

$$
\begin{gathered}
d_{k}=\left(d_{k}-c_{k}\right)+c_{k}, \quad d_{k}-1=\left(d_{k}-1-c_{k}\right)+c_{k}, \\
-2 c_{k-1} \leq d_{k}-1-c_{k}<d_{k}-c_{k}<-c_{k}+d_{k-1}
\end{gathered}
$$

it follows that $d_{k}-c_{k}, d_{k}-1-c_{k} \in W_{k}$ and then $d_{k}, d_{k}-1 \in W_{k}+\left\{c_{k}\right\}$. Hence $d_{k+1} \leq d_{k}-2$. By (iv), we have $w_{j+1}-w_{j} \in\{1,2\}$. Since $d_{k} \rightarrow-\infty$, by (ii) we have $(-\infty, 9] \subseteq W+C$. For any integer $n \geq 10$, there exists an $i$ such that $-c_{i-1} \leq n<-c_{i}$. Hence

$$
-c_{i}+d_{1}<-c_{i-1}-c_{i} \leq n-c_{i}<-2 c_{i}
$$

and so $n-c_{i} \in W_{i}$, that is, $n \in W_{i}+\left\{c_{i}\right\}$. Therefore, $W+C=\mathbb{Z}$. 
Next, we prove that the complement $C$ is minimal. For any positive integer $i$, we write $d_{i}=c+w$ with $c \in C$ and $w \in W$. Now we shall prove that $c=c_{i}$. By (iv), we have $d_{i}-c_{j} \notin W$ for all integers $j>i$. Hence $c \neq c_{j}$ for all integers $j>i$. Since $-2 c_{i-1}$ is the minimal value of $W \backslash W_{i-1}$ and for any positive integers $j \leq i-1, d_{i}-c_{j} \leq d_{i}-c_{i-1}<-2 c_{i-1}$, it follows that $d_{i}-c_{j} \notin W \backslash W_{i-1}$ for all positive integer $j \leq i-1$. Noting that $d_{i} \notin W_{i-1}+\left\{c_{1}, \ldots, c_{i-1}\right\}$, we have $d_{i} \notin W+\left\{c_{1}, c_{2}, \ldots, c_{i-1}\right\}$. Hence $c=c_{i}$.

Therefore, $C$ is a minimal complement to $W$. Furthermore, by (iii), we can choose suitable $c_{i}$ such that $W$ is infinite and not eventually periodic.

\section{Acknowledgement}

This work was done during the third author visiting to Budapest University of Technology and Economics. He would like to thank Dr. Sándor Kiss and Dr. Csaba Sándor for their warm hospitality. We would liketo thank the anonymous referee very much for the detailed comments.

$\Rightarrow$

manumbersase Supported by the ÚNKP-18-4 New National Excellence Program of the Ministry of Human Capacities.

\section{References}

[1] Y.-G. Chen, J.-H. Fang, On additive complements, II, Proc. Amer. Math. Soc. 139 (3) (2011) 881-883.

[2] Y.-G. Chen, Q.-H. Yang, On a problem of Nathanson related to minimal additive complements SIAM J. Discrete Math. 26 (4) (2012) 1532-1536.

[3] J.-H. Fang, Y.-G. Chen, On additive complements, Proc. Amer. Math. Soc. 138 (6) (2010) 1923-1927. 
[4] M.-B. Nathanson, Problems in additive number theory, IV: Nets in groups and shortest length g-adic representations, Int. J. Number Theory 7 (3) (2011) 1999-2017.

[5] I. Z. Ruzsa, On the additive completion of linear recurrence sequences, Periodica Math. Hungar 9 (1978) 285-291.

[6] I. Z. Ruzsa, Additive completion of lacunary sequences, Combinatorica 21 (2) (2001) 279-291. 